\title{
Chemical industrial areas and their dynamic danger behaviour
}

\author{
G. L. L. Reniers, A. Audenaert, W. Dullaert \& K. Soudan \\ Antwerp Research Group on Safety and Security ARGoSS, \\ University of Antwerp, Prinsstraat 13, 2000 Antwerp, Belgium
}

\begin{abstract}
Chemical industrial areas or so-called chemical clusters consist of various companies situated next to each other. Such areas are composed of hundreds of chemical installations exhibiting danger to a certain degree for initiating or continuing knock-on effects. In this paper, a methodology to model such clusters as networks and to investigate their dynamic danger behaviour is proposed. This original approach leads to a tentative strategy to optimally protect chemical industrial areas against security risks.

Keywords: small-world behaviour, power-law functioning, network analysis, security management.
\end{abstract}

\section{Introduction}

The emerging importance of so-called scale-free networks is a direct consequence of the widespread and multidisciplinary real-life occurrence of such networks, e.g. the world-wide web, social networks, terrorist networks, etc. (Barabasi [1]; Barabasi et al. [2], Buchanan [3]; Gong and Van Leeuwen [4]). In this paper the importance of such networks for the security management research field is examined.

To diagnose a network as scale-free, it has to be typified by three main characteristics, i.e., (i) every pair of nodes can be connected through a short path within the network, (ii) the probability that two nodes are linked is greater if they share a neighbour, and (iii) the fraction of nodes with $k$ neighbours decays roughly as a function of the form $k^{-\gamma}$ for some positive exponent $\gamma$. 
A network satisfying only features (i) and (ii) is called a small-world network (Watts [5]). The third characteristic, so typical of scale-free networks, can be defined as the network obeying a power-law distribution in the number of connections between nodes in the network, i.e., very few nodes exhibit very high connectivity (such nodes are generally called 'hubs') while the vast majority of nodes have only few links.

As ever more networks are identified to be scale-free the question arises whether an industrial area composed of hundreds of chemical installations might be considered as a scale-free network. If so, chemical clusters can be protected in a more rational way.

To obtain an understanding of the real intentional risks in the chemical process industry, investigating the dynamic danger behaviour of chemical surroundings (which can be viewed as networks) might offer some valuable insights. A more mathematical approach to carrying out security vulnerability assessments in an industrial area might be appropriate. A new management perspective would indeed be needed in a chemical industrial area as regards the protection of chemical equipment.

\section{Research objectives}

It must be borne in mind that if safety and security management fails to systematically carry out analyses and assessments to identify and to evaluate an installation's safety and security risks, its danger towards the other installations in the area automatically (over time) increases. The latter can be referred to as the dynamic behaviour of a chemical installation. To study the dynamic properties of an industrial area, the area needs to be modelled as a network of installations. The consequences of such dynamic features for the network depend largely on the network topology and its structure. In order to address these issues, this paper investigates three main research questions to gain a better understanding of the complex environment of a chemical cluster and two secondary, more practical, questions (indicated by 'A') following the main questions:

(i) Can an industrial area be modelled as a structured network, i.e., a directed weighted graph?

(iA) How can an industrial area be more optimally protected?

(ii) Can an industrial area be considered as a small-world?

(iii) Does an industrial area display scale-free characteristics?

(iiiA) How can a scale-free industrial area be more optimally protected?

Answering these research questions requires obtaining a holistic view of a chemical industrial area. How the latter can be achieved is explained in the next section. 


\section{A chemical industrial area network}

An industrial region consists of a number of separate chemical installations. Every chemical installation has an intrinsic hazard depending on the amount of substances present, the physical and toxic properties of the substances and the specific process conditions. Hence, such installations present - to a greater or lesser extent - a danger to their environment. In this way installations are mutually linked in terms of the level of danger they pose to each other.

In this particular research on the dynamics of an industrial area, installations are considered to be the nodes of a network and the amount of danger between the installations is represented by the edges linking the nodes together. Using this approach, a chemical industrial area can be modelled as a connected weighted network.

In many applications involving graphs, it is useful to introduce a variable that represents the weight of each arc, like for example, the arc cost or the arc distance. In this research, the weight of an $\operatorname{arc}\left(v_{i}, v_{j}\right)$ with $v_{i} \neq v_{j}$ represents the amount of danger outgoing from installation $v_{i}$ onto installation $v_{j}$. In our case of investigating domino effects, mathematically the arc weight is simply a scalar (real number) referred to as the Domino Danger Unit ( $D D U)$. Let $D D U_{i j}$ denote the weight of an arc $\left(v_{i}, v_{j}\right)$ with $v_{i} \neq v_{j}$, such that $D D U_{i j} \in R^{+}$if $v_{i} \neq v_{j}$ and $D D U_{i j}=0$ if $v_{i}=v_{j}$. By calculating all (unidirectional) Domino Danger Units between all nodes in the entire network we obtain an installations danger matrix $\boldsymbol{D D U}$ of order $n \times n$. The factor $D D U_{i j}$ is a measure of the danger that installation $v_{i}$ represents for installation $v_{j}$ in terms of domino effects. The Domino Danger Unit $D D U_{i j}$ is calculated between every node $v_{i}$ and the remaining nodes $v_{j}\left(v_{j} \in G ; v_{j} \neq v_{i}\right)$ in the graph $G(N, A)$ representing the installations network with $N$ the number of nodes and $A$ the number of arcs within the graph, implying that $D D U_{i j}$ does not have to be equal to $D D U_{j i}$, although the possibility exists.

A total danger $T D_{i}$ for every node $v_{i}$ can be calculated by summing the $D D U_{i j}$ :

$$
T D_{i}=\sum_{j(\neq i) \in G} D D U_{i j}
$$

A more thorough discussion about the tool which was developed to carry out the procedure described above, can be found in Reniers and Dullaert [6,7]. 


\section{Definitions and notations}

The mathematical translation of the three characteristics defining a scale-free network as explained in the introductory section, is based on the evaluation of three quantities, i.e., the characteristic path length

$$
L=\frac{1}{N(N-1)} \sum_{i \neq j} d_{i j},
$$

the clustering coefficient

$$
C=\frac{1}{N} \sum_{i} C_{i},
$$

and the power-law degree (the degree of a vertex is the total number of its outgoing links) distribution $P(k) \sim k^{-\gamma}$. It should be noted that $\boldsymbol{d}$ is the matrix of the shortest path weights (the smallest sum of the weights on the edges throughout all the possible paths in the graph, whatever the connotation of the weights) between two generic vertices $v_{i}$ and $v_{j} . C_{i}$ is the number of edges existing in $G_{i}$, the subgraph of the connected neighbours of $v_{i}$, divided by the maximum possible number $k_{i}\left(k_{i}-1\right) / 2$. $k_{i}$ is the number of edges originating from vertex $v_{i}$.

Small-world networks are highly clustered and possess small characteristic path lengths. Small-world networks, which are scale-free, have a distribution of the number of connections of a generic vertex $v_{i}$ following a power-law. To deal with weighted networks Latora and Marchiori [8] introduce the 'Efficiency' parameter $E$ of a network. Efficiency allows for small-world networks to be seen as systems that are both globally and locally efficient. $1 / L$ and $C$ can be seen as first approximations of $E$, evaluated on a global and a local scale respectively.

As already mentioned, the domino threat that every vertex (installation) poses to every other vertex (installation) in the network can be represented by the Domino Danger Unit associated with the directed edge between every couple of vertices.

On the one hand, the efficiency $\varepsilon_{i j}$ of our network is then assumed to be proportional to the aggregated danger of the path between two nodes $v_{i}$ and $v_{j}$. On the other hand, the number of links is inversely proportional to the efficiency $\varepsilon_{i j}$. The more links there are to get from one installation to another in the network, the less dangerous the network is, thus the lower the efficiency according to the measures of Latora and Marchiori.

The 'direct efficiency' can then be calculated by considering the path in a network between two nodes $v_{i}$ and $v_{j}$ which is characterized by the lowest number of steps to go from $v_{i}$ to $v_{j}$. Hence, the direct efficiency can be expressed in mathematical terms as follows: 


$$
\varepsilon_{i j}^{\text {direct }}=\underset{p a t h i \rightarrow j}{\operatorname{Min}}\left(\frac{\sum_{i^{\prime} \neq j^{\prime} \in G} D D U_{i^{\prime} j^{\prime}}}{A_{s}^{i j}}\right), \forall v_{i}, v_{j}
$$

with $A_{s}^{i j}=$ number of links of the path going from $v_{i}$ to $v_{j}$, and having the lowest number of links. It is assumed that $A_{s}^{i j} \geq 1$. If no path exists between $v_{i}$ and $v_{j}$, $A_{s}^{i j}=+\infty$ and thus consistently $\varepsilon_{i j}=0$.

We can also determine the 'indirect efficiency' of the network by calculating the maximum aggregated danger of the path between two nodes $v_{i}$ and $v_{j}$. To this end, a path with highest aggregated weight between the nodes $v_{i}$ and $v_{j}$ (and using the Domino Danger Units as weights) for every couple of nodes in the graph, has to be constructed. Since the number of links of the longest path (from node $v_{i}$ to node $v_{j}$ having the highest aggregated danger), $A_{l}^{i j}$, is inversely proportional to the efficiency $\varepsilon_{i j}$, this factor also has to be taken into account. Hence, the indirect efficiency can be expressed in mathematical terms as follows:

$$
\varepsilon_{i j}^{\text {indirect }}=\underset{\text { pathi } \rightarrow j}{\operatorname{aax}}\left(\frac{\sum_{i^{\prime} \neq j^{\prime} \in G} D D U_{i^{\prime} j^{\prime}}}{A_{l}^{i j}}\right), \forall v_{i}, v_{j}
$$

with $A_{l}^{i j}=$ number of links of the path going from $v_{i}$ to $v_{j}$, and having the highest aggregated danger. It is assumed that $A_{l}^{i j} \geq 1$. If no path exists between $v_{i}$ and $v_{j}$, $A_{l}^{i j}=+\infty$ and thus consistently $\varepsilon_{i j}=0$.

The average efficiency of graph $G$ can be defined as:

$$
E(G)=\frac{\sum_{i \neq j \in G} \varepsilon_{i j}}{N(N-1)}
$$

The global efficiency $E_{\text {glob }}$ of $G$ is the efficiency of the entire network and can be defined as:

$$
E_{\text {glob }}=\frac{E\left(G^{\text {direct }}\right)}{E\left(G^{\text {indirect }}\right)}
$$

A high global efficiency is consistent with a low characteristic path length. Since the efficiency can also be evaluated for any subgraph of $G$, it can be used to 
evaluate the local properties of the graph. Therefore, using the efficiencies for every graph $G_{i}\left(G_{i}\right.$ is the subgraph of the neighbours of $v_{i}$ ( $v_{i}$ not included). A neighbour of $v_{i}$ is a node (i.e., a chemical installation) vulnerable to (receiving domino danger from) installation $I$ ), the local efficiency of $G$ can be expressed:

$$
E_{l o c}(G)=\frac{1}{N} \sum_{i \in G} \frac{E\left(G_{i}^{\text {direct }}\right)}{E\left(G_{i}^{\text {indirect }}\right)}
$$

with:

$$
E\left(G_{i}^{\text {direct }}\right)=\frac{1}{k_{i}\left(k_{i}-1\right)} \sum_{l \neq m \in G_{i}} \operatorname{Min}_{p a t h l \rightarrow m}\left(\frac{\sum_{i^{\prime} \neq j^{\prime} \in G_{i}} D D U_{i^{\prime} j^{\prime}}}{A_{S}^{l m}}\right)
$$

and:

$$
E\left(G_{i}^{\text {indirect }}\right)=\frac{1}{k_{i}\left(k_{i}-1\right)} \sum_{l \neq m \in G_{i}} \operatorname{Maxhl}_{l}\left(\frac{\sum_{i^{\prime} \neq j^{\prime} \in G_{i}} D D U_{i^{\prime} j^{\prime}}}{A_{l}^{l m}}\right)
$$

Since $v_{i} \notin G_{i}, E_{l o c}$ indicates the fault tolerance of the system, i.e., the efficiency of the domino danger spreading between the first neighbours of $v_{i}$ when $v_{i}$ is removed.

In general, a network $G(N, A)$ representing an industrial chemical area can then be identified as being a small-world if it gives rise to both a high $E_{g l o b}$ and a high $E_{l o c}$, such a network thus proving efficient both in global and local domino danger spreading.

Summarizing research question (ii), i.e., the small-world features of the cluster network, the industrial area is considered as a graph $G(N, A)$ and is represented by its connection matrix $\boldsymbol{a}$ ( $\boldsymbol{a}$ is the $N \mathrm{x} N$ matrix where $a_{i j}=1$ if there exists an edge $\left(v_{i}, v_{j}\right)$ and $a_{i j}=0$ otherwise) and by its Domino Danger Units matrix $\boldsymbol{D D} \boldsymbol{U}$. The matrix of the longest path weights can then be calculated by using the information contained both in $\boldsymbol{a}$ and $\boldsymbol{D D} \boldsymbol{U}$. Subsequently, code is used to determine the global efficiency and the local efficiency of the network. If both calculated efficiencies return high values, the network (i.e., the chemical installations cluster) displays small-world behavior. First, the network is only $\left(100-E_{\text {glob }}\right) \%$ less efficient in propagating domino effects than the ideal network with a danger link from each installation to the others. Second, a high local 
efficiency of $E_{l o c}$ indicates that the site is very "fault tolerant": removing an installation chosen at random in the area, and all the danger links with it, will not dramatically affect the efficiency in the danger linking between the neighbours of that installation.

Another point of interest is research question (iii), which can be tackled by investigating the power-law distribution of the danger connection topology of the network(s). The installations situated on an industrial area (or sub-area) might be characterized by an uneven distribution of danger connectedness. Some (very dangerous) installations then act as "highly connected" hubs for initiating or continuing domino effects. This might dramatically influence the way the industrial area network breaks down. Hence, to determine whether an industrial area is scale-free, the dangers linking the installations to a network have to follow a power-law distribution. To investigate the latter in a directed weighted graph, the total domino danger $T D_{i}$ of a vertex is used to obtain a vertex "outstrength distribution". In the case of an industrial area, the automatic increase of the $T D_{i}$ (if no risk analyses are carried out and no resulting precaution and protection measures are taken), has a higher impact on already dangerous installations since their domino danger increases towards a lot of nearby installations. Therefore, a network $G(N, A)$ representing an industrial chemical area can be identified as being scale-free if it is typified by an out-strength distribution following a power-law. This leads to finding an answer to research question (iiiA), which is discussed in the next section.

\section{Proposed methodology and results}

As already mentioned, building a user-friendly software tool for investigating knock-on effects related to safety risks as well as security risks requires viewing a chemical cluster in a holistic way. Domino effect investigations should be carried out regardless of company borders and perimeters.

Once the area is characterized by a single network of installations, it can be divided into smaller networks, i.e., smaller 'clustered chemical installations', where there are no links between the sub-clusters. Such sub-clusters can be called domino islands. This can be achieved by eliminating those installations (nodes) exhibiting the largest $T D$-values (e.g., the top 3\%). (There exists no correlation whatsoever between installation failure probabilities and terrorist attacks. Therefore, when splitting the cluster area into several sub-clusters, no failure probabilities should be taken into account. Hence, only probabilityindependent consequences of domino effect scenarios serve as guiding parameters to divide the cluster into smaller areas.) The latter approach makes the region much less vulnerable to terrorist threats since the area can no longer be destroyed in one go; only the sub-clusters might break down entirely. Figure 1 illustrates dividing an area (originally vulnerable when broken down as a whole) into smaller areas leading to domino islands between which no domino effects can occur.

Simulations carried out by the authors on a real industrial area indicate that by eliminating as less as $1 \%$ (3\%respectively) of the domino danger hubs in an area, 
about $20 \%$ (30\% respectively) of the total domino danger and about $12 \%(20 \%$ respectively) of the number of links are simultaneously eliminated as well. Moreover, the whole area is divided into several domino islands where no domino effects can occur in between. Further removing domino danger hubs in the area reveals additional domino islands and further decreases the number of links and the total domino danger, compared with the original network. The higher the percentage of hubs removed, the lower the additional reduction of domino links and of the total domino danger. Hence, a trade-off has to be made between security efforts (costs) and the preferred number of emerged domino islands.

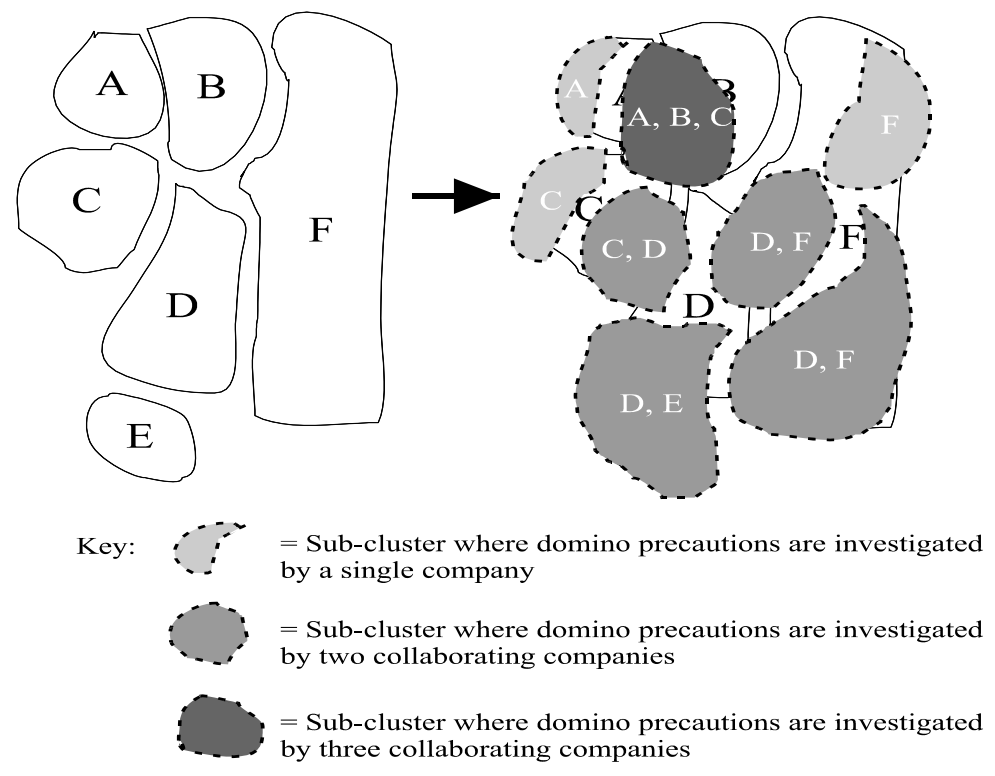

Figure 1: Example of an industrial area comprising six plants $(A, B, C, D, E$, and $F$ ) divided into sub-clusters.

Afterwards, global and local efficiencies of the domino islands need to be investigated. Results indicate domino islands may display small-world behaviour characterized by high global and local efficiencies. For the areas where the latter is the case, an investigation towards their scale-free behavior should be carried out. All different neighbouring companies belonging to the sub-clusters need to collaborate with each other to make those installations acting as "domino effect hubs" more safe. If the domino island ceases to exhibit scale-free behavior, the area can truly be considered more secured against intentional attacks.

\section{Conclusions}

Evaluating domino effect risks is not at all an easy task. Moreover, assessing domino security risks requires another approach than estimating domino safety 
risks. The mathematical requirements and the working procedure for actually efficient and effective taking security precautions in an industrial area are elaborated in this paper.

A cluster is first constructed as a network of chemical installations, linked with danger edges. Second, by calculating the global and local efficiencies of danger spreading within the area, it is investigated whether the area acts as a small-world. Third, the cluster's scale-free behaviour is examined. Based on these analyses, security measures can be taken leading to a substantial decrease of domino danger spreading.

\section{References}

[1] Barabasi, A.-L., Linked. How everything is connected to everything else and what it means for business, science and everyday life. Plume, New York, 2003.

[2] Barabasi, A.-L., Albert, R., Jeong, H., Scale-free characteristics of random networks: the topology of the world-wide web, Physica A, 281, pp. 69-77, 2000.

[3] Buchanan, M., Nexus. Small worlds and the groundbreaking science of networks, W.W. Norton and Company, New York, 2003.

[4] Gong, P., Van Leeuwen, C., Emergence of scale-free network with chaotic units, Physica A, 321, pp. 679-688, 2003.

[5] Watts, D.J., Small Worlds, Princeton University Press, New Jersey, 2004.

[6] Reniers, G.L.L., Dullaert, W., DomPrevPlanning: user-friendly software for planning domino effects prevention, forthcoming in (2007a): Safety Science.

[7] Reniers, G.L.L., Dullaert, W., Knock-on accident prevention in a chemical cluster, forthcoming in (2007b): Expert Systems With Applications.

[8] Latora, V., Marchiori, M., Economic small-world behaviour in weighted networks, Eur Phys. J. B 32, pp. 249-263, 2003. 